\title{
A Survey of Electron Microscopic Methods for 3-D Imaging
}

\author{
Michael Marko
}

Resource for Visualization of Biological Complexity, Wadsworth Center, Empire State Plaza, Albany, NY 12201-0509

There are three basic methods for obtaining 3-D images using electron microscopy: (1) recording two views of the specimen to form a stereo pair image, (2) recording many views to compute a 3-D reconstruction, and (3) imaging serial depth planes to build a 3-D volume.

Conventional SEM images have shading cues that we naturally interpret as depth, but without careful consideration of SEM image formation, we may be misled [1]. In the case of specimens that are metal-shadowed at a known angle, height may be determined by the length of the shadow. For general applications, reliable depth information may be obtained from a stereo pair of images, obtained by tilting the specimen. Because of the horizontal axis of our eyes, the images must be viewed with the tilt axis vertical. Then, height may be determined from parallax, which is the relative $\mathrm{x}$-axis displacement of a corresponding feature in the two images. If we assume that we are able to see only one surface at any point in an SEM image, a 3-D topographical map may be made using parallax to generate the third dimension. Commercial software is available to accomplish this [2]. The resolution in depth, as measured by parallax, may in principle be equal to the original image resolution.

Serial depth images may be recorded in the SEM by cutting away a thin layer of the specimen, recording an image, and then cutting away another layer. The most common way to remove the layers is by ion milling in a dual-beam FIB/SEM. This technology is rapidly being adopted in both materials science and biology $[3,4,5]$. The resolution in depth may be about $20 \mathrm{~nm}$ with "FIB tomography". An alternative approach is to place a microtome inside the SEM and use a diamond knife to cut away the layers [6]. The depth resolution with this method may be $50 \mathrm{~nm}$, and the inplane resolution is about $10 \mathrm{~nm}$. These "serial block face" techniques are particularly useful for reconstructing large volumes of material, and avoid the concerns about sectioning distortion and section-to-section alignment that come from imaging sections after they are cut.

In STEM, beam convergence may be increased in order to minimize the depth of the specimen that is in sharp focus. In the scanning confocal electron microscope, structure of internal features may be studied in specimens up to 8-10 $\mu \mathrm{m}$ thick, with a lateral resolution of $10-100 \mathrm{~nm}$ depending on specimen thickness $[7,8]$. With aberration-corrected STEM, very high convergence angles can be used, and subnanometer depth resolution may be expected in the future. At present, with corrected STEM, lateral resolution in the one-Ấngström range is accompanied by depth resolution of about $4 \mathrm{~nm}[9,10]$.

In TEM, stereo-pair imaging has been used since the earliest days. It became especially popular in the 1970s when high-voltage electron microscopes (HVEM) were being installed in several laboratories. In TEM, the specimen is translucent, so unlike SEM (or our normal visual environment), in HVEM we look through many overlapping surfaces, all of which are in sharp focus. Without stereo viewing, it is nearly impossible to understand many HVEM images of thick specimens. Software was developed to make contour-based 3-D reconstructions from stereo pairs of serial thick sections [11]. This method was superior to conventional TEM serial thin reconstruction, although the discomfort of prolonged viewing of "unnatural" stereo made it unpopular. The depth resolution is limited to about $50 \mathrm{~nm}$ for 
either method, but for different reasons. Development of software for 3-D analysis based on stereopairs continued, and has proven to be useful in TEM and SEM [12].

At present, most 3-D TEM imaging is done using either electron tomography $[13,14]$ or the "single particle" approach [15], both of which involve recording many views of a specimen and computing a 3-D volume or "map" of an object. Electron tomography is suited for reconstruction of individual, unique objects, since all views are recorded from the same object. For thin materials science specimens that can tolerate the high electron dose required, depth resolution as high as $1 \mathrm{~nm}$ may be achieved. For dose-sensitive "native" (frozen-hydrated) biological specimens, the depth resolution is limited to about $4 \mathrm{~nm}$ at present. High-resolution 3-D imaging in biology relies on the "single particle" approach, in which (in its simple form) it is assumed that each particle, for example a macromolecule or virus, is identical and randomly oriented in the image. A field containing many particles need be imaged only once, and the particles are classified according to orientation so that a reconstruction can be computed. In many cases, the particles have symmetry, often helical or icosahedral, which reduces the number of particles (images) needed for a given resolution. Up to $10^{5}$ particles are typically required. 3 -D resolution on the order of $10-15 \AA$ is typical at present, but 3$4 \AA$ has been achieved with some specimens. A special case of 3-D imaging is electron diffraction of crystals, which is capable of atomic or near-atomic resolution in both materials science and biology [16].

Increasingly, we wish to combine 3-D imaging with 3-D elemental mapping. Stereo-pairs of elemental maps may be made using EFTEM, EDX, or EELS in the analytical TEM. Such maps may in some cases be fitted to conventional tomograms [17]. Tomographic reconstructions may be computed from elemental maps acquired by STEM spectrum imaging (EDX or EELS), or EFTEM, and there have been applications in both materials science [18,19] and biology [20]. [21]

References

[1] J.I. Goldstein, et al., Scanning Electron Microscopy and X-ray Microanalysis, $3^{\text {rd }}$ edition, Kluwer, 2002.

[2] http://www.alicona.com/

[3] A.J. Kubis et al. Metall Mater. Trans. A. 35a (2004) 1935.

[4] R. Williams et al. Microsc. Microanal. 10 (Suppl. 2) (2004) 1178CD.

[5] I. Gestmann et al. Microsc. Microanal. 10 (Suppl. 2) (2004) 1124CD.

[6] W. Denk and H. Horstmann. PLOS Biology 2 (2004) 1900.

[7] N.J. Zaluzec Microscopy Today 11(6) (2003) 18.

[8] S.P. Frigo et al. Applied Physics Letters 81 (2002) 2112.

[9] Y. Peng et al. Microsc. Microanal. 10 (Suppl. 2) (2004) 1200CD.

[10] S.J. Pennycook et al. Microsc. Microanal. 10 (Suppl. 2) (2004) 1172CD.

[11] M. Marko and A. Leith J. Elect. Microsc. Tech. 9 (1988) 395.

[12] M. Marko and A. Leith. J. Struct. Biol. 116 (1996) 93.

[13] J. Frank, Electron Tomography, Plenum, 1992.

[14] A.J. Koster et al., J. Struct. Biol. 120 (1997) 276.

[15] J. Frank, Three-dimensional Electron Microscopy of Macromolecules, Academic Press, 1996.

[16] J.M. Cowley, Diffraction Physics $3^{\text {rd }}$ Edition, Elsevier, 1995.

[17] M. Marko et al., Microscopy Today, 11(6) (2002) 14.

[18] P.A. Midgley and M. Weyland, Ultramicroscopy, 96 (2003) 413.

[19] G. Möbus et al. Ultramicroscopy 96 (2002) 433.

[20] R.D. Leapman et al. Ultramicroscopy 100 (2004) 115.

[21] Supported by NIH / NCRR Biomedical Research Technology Program Grant RR01219 (PI J. Frank). 